\title{
FLUID SPACE MEASUREMENTS IN BURNED CHILDREN
}

\author{
BY \\ P. K. MÄKELÄ* and K. DIXON \\ From the Burns Unit and Pathology Laboratory, Booth Hall Children's Hospital, Manchester
}

(RECEIVED FOR PUBLICATION DECEMBER 3, 1962)

Fluid imbalance, caused by fluid loss and changes in capillary permeability, occurs during the acute phase of burns and scalds, and the extent of the loss has been shown to depend on the body weight and surface area damaged. The blister fluid may contain protein in a concentration up to $6 \mathrm{~g} . / 100 \mathrm{ml}$. and sodium up to $160 \mathrm{mEq} / \mathrm{l}$., resembling lymph and interstitial fluid in the burned patient (Cope and Moore, 1944; Sevitt, 1957). There is a generalized increase in capillary permeability, resulting in a loss of protein from the plasma, principally of albumin (Cope and Moore, 1944; Cope, Graham, Moore and Ball, 1948; Bull, 1958), and a consequent fall in blood volume with movement of fluid into the interstitial space. Factors responsible for this change in permeability have been described by Miles and Wilhelm (1960) and Sevitt (1957). Fluid transfer ceases by the end of the second post-burn day, and Cope and Moore (1944) have shown that normal capillary permeability to radioactive dyes and proteins is restored within $\mathbf{4 0}$ hours after burn injury.

Using thiocyanate ion and $\mathrm{Na}^{24}$, Cope and Moore (1947) found an increase in extracellular space in burned patients which was maximal in 36 to 48 hours. In contrast, Fogelman and Wilson (1955), in studies of scalded dogs and adult burned patients, found a decrease in extracellular space as measured by $\mathrm{S}^{35} \mathrm{O}_{4}$, while the total body water remained unchanged. In support of this, the investigations of local thermal oedema by Rosenthal and Tabor (1945) in man, and by Fox and Baer (1947) in mice, indicate that sodium retention takes place in injured cells, resulting in a fall in plasma concentration accompanied by extracellular dehydration. In order to obtain more information into the distribution of fluid spaces in burned and scalded children experiments were performed to measure total body water and extracellular space, using antipyrine and sodium thiosulphate respectively. The total extracellular sodium was calculated by reference to serum concentration.

\section{Material and Method}

Antipyrine and thiosulphate spaces have in the past usually been estimated separately, but we have found that it is possible to perform these measurements simultaneously.

The procedure has been carried out on 60 occasions, on convalescent and critically burned patients without demonstrable side-effects, whereas when the thiosulphate experiment is carried out alone nausea is a frequent complication.

An intravenous nylon cannula was used to take blood samples and also to introduce the solution of sodium thiosulphate and antipyrine. A slow infusion of $5 \%$ dextrose maintained patency of the cannula at other times. After taking a blank sample of blood, equal volumes of $2 \%$ antipyrine solution (prepared in the hospital pharmacy) and $10 \%$ sodium thiosulphate (B.D.H.) were mixed. An injection of $2 \mathrm{ml} . / \mathrm{kg}$. body weight was started at zero time and given in 15 minutes. Eight 1-2 ml. samples of blood were taken, without stasis, into heparinized tubes, and the plasma separated immediately by centrifugation. The first six samples, taken between 20 and 70 minutes after the beginning of the injection, were analysed for thiosulphate. The last two samples were taken 90 and 110 minutes after the beginning of the injection. All samples taken more than $\mathbf{4 0}$ minutes after the beginning of the injection were assayed for antipyrine. A portion of the injection solution was used as a standard and diluted $1: 125$ for thiosulphate, and 1:250 for antipyrine analysis. using the same quantities and pipettes as for the appropriate ultrafiltrate and plasma samples.

Experiments were performed on plasma to which had been added antipyrine $(0-100 \mu \mathrm{g} . / \mathrm{ml}$.) and sodium thiosulphate $(0-500 \mu \mathrm{g} . / \mathrm{ml}$.$) , and the results are sum-$ marized in Table 1.

The average recovery of antipyrine was $97.7 \%$ (standard error of the mean $\pm 1.1 \%$ ), and of thiosulphate $106 \cdot 7 \%$ (standard error of the mean $\pm 1 \cdot 5 \%$ ). The latter result was not significantly different from the theoretical recovery expected in plasma water, $\left.\left(\frac{100}{1-\text { protein fraction }}=107 \%\right)\right)$. 
In the concentrations used, determinations of antipyrine and of thiosulphate were unaffected by the presence of the other component.

Initial determinations were carried out on two subjects and the results are shown in Table 2.

Investigations were performed on 16 patients admitted to the burns unit of this children's hospital. Their ages ranged from 18 months to 10 years, their injuries involved from 4 to $24 \%$ of the total body surface area, and all recovered. During the acute phase the more seriously injured children were resuscitated with intravenous and oral fluid therapy. Minor burns and scalds were maintained on a normal diet, but received supplementary oral fluids to cover anticipated excessive losses. In each case measurements were carried out 46-48 hours after injury, and repeated during convalescence.

Venous packed cell volume (P.C.V.) was determined by centrifugation at $5,000 \mathrm{~g}$. for five minutes. Plasma proteins were determined by the biuret method, and plasma sodium and potassium concentration by flame photometry. The single injection method for simultaneous measurement of antipyrine (total body water) and thiosulphate dilution (extracellular water) spaces was carried out as described above, the analytical procedures being based on the antipyrine method of Mendelson and Levin (1960) and the thiosulphate method of Newman, Gilman and Philips (1946) (see Appendix). The intracellular water was obtained by difference between extracellular and total body wacer volumes. The relative plasma volume was calculated from the blood P.C.V. Red cell destruction during the first two days is minimal in thermal injuries of this extent (Muir and Stewart, 1959), and was ignored.

\section{Summary of Cases}

A. Burns Receiving Specific Fluid Resuscitation Therapy

Case 1. An 8-year-old girl sustained a partial and full thickness burn involving breast, abdomen and both thighs $(20-22 \%$ body surface area) when her clothes were ignited. Plasma, $450 \mathrm{ml}$., glucose-saline, $450 \mathrm{ml}$., and Hartmann's solution, $450 \mathrm{ml}$., were given intravenously during the first day. The urine output was, however, unsatisfactory and the P.C.V. rose to $50 \%$. During the second day the estimated requirement of plasma $(450 \mathrm{ml}$.) was given, but when the measurements wele performed 46 hours after burning the P.C.V. was $47 \%$, indicating a plasma volume which was still only $85 \%$ of control. (By 'control' is meant the corresponding value for the same patient at the time of the second (convalescent) determination.) The clinical condition of the patient was, however, satisfactory. There was a comparable fall in the three parameters, extracellular water, total body water and intracellular water, and a more marked fall in the extracellular sodium.

Case 2. A girl of 5 years received $15 \%$ burns, including full and partial thickness skin loss of the abdomen and thighs. The intravenous therapy consisted of $300 \mathrm{ml}$. plasma and $100 \mathrm{ml}$. Hartmann's solution. After 24 hours the urine output was satisfactory
TABLE 1

RECOVERY OF ANTIPYRINE AND THIOSULPHATE ADDED TO PLASMA

\begin{tabular}{|c|c|c|c|c|c|}
\hline & & $\underset{(\mu \mathrm{g} . / \mathrm{ml} .)}{\text { Range }}$ & $\begin{array}{l}\text { No. of } \\
\text { Samples }\end{array}$ & $\begin{array}{c}\% \\
\text { Recovery }\end{array}$ & $\begin{array}{c}\text { Standard } \\
\text { Error } \\
\text { of Mean } \\
(\%)\end{array}$ \\
\hline $\begin{array}{l}\text { Antipyrine } \\
\text { Thiosulphate }\end{array}$ & $\begin{array}{l}\cdots \\
\cdots\end{array}$ & $\begin{array}{l}0-100 \\
0-500\end{array}$ & $\begin{array}{l}12 \\
14\end{array}$ & $\begin{array}{r}97 \cdot 7 \\
106 \cdot 7\end{array}$ & $\begin{array}{l} \pm 1 \cdot 1 \\
\pm 1 \cdot 5\end{array}$ \\
\hline
\end{tabular}

TABLE 2

RESULTS OBTAINED FROM TWO CONTROL HOSPITAL PATIENTS

\begin{tabular}{|c|c|c|c|c|c|}
\hline \multirow{2}{*}{ Case } & \multirow{2}{*}{$\begin{array}{c}\text { Age } \\
\text { (yrs) }\end{array}$} & \multicolumn{2}{|c|}{ Thiosulphate Space } & \multicolumn{2}{|c|}{ Antipyrine Space } \\
\hline & & $\begin{array}{l}\text { (ml. } / 100 \mathrm{~g} . \\
\text { body } \mathrm{wt} .)\end{array}$ & $(1 . / \mathrm{m} \cdot 2)$ & $\begin{array}{l}\text { (ml./100 } \mathrm{g} . \\
\text { body wt.) }\end{array}$ & $(1 . / \mathrm{m} .2)$ \\
\hline $\begin{array}{l}1 \\
2\end{array}$ & $\begin{array}{l}5 \\
6\end{array}$ & $\begin{array}{l}14 \cdot 5 \pm 0 \cdot 5 \\
21 \cdot 1 \pm 0 \cdot 3\end{array}$ & $\begin{array}{l}3 \cdot 8 \pm 0 \cdot 1 \\
5 \cdot 04 \pm 0 \cdot 07\end{array}$ & $\begin{array}{l}62 \pm 1 \\
59 \pm 2\end{array}$ & $\begin{array}{l}16 \cdot 3 \pm 0 \cdot 3 \\
13 \cdot 9 \pm 0 \cdot 4\end{array}$ \\
\hline
\end{tabular}

Following the experimental value is the standard error of the mean, obtained from a single experiment and calculated from the deviation of the experimental points from the regression line.

and the P.C.V. was $49 \%$. During the second day intravenous therapy was continued with $200 \mathrm{ml}$. plasma. At the time of the initial experiment the P.C.V. had fallen to $43 \%$ (i.e. plasma volume $89 \%$ of the control), and the patient was in a satisfactory clinical state. The extracellular water determination was unsuccessful. Total body water was not significantly altered.

Case 3. A 10-year-old girl sustained $10 \%$ burns of gluteal region and back of thighs following a nightdress fire. Approximately half of the area had suffered full thickness destruction. Intravenous fluid therapy became necessary after 24 hours, when the P.C.V. had risen to $60 \%$. The pulse was rapid and the girl was restless and vomiting. After four hours' treatment vomiting had ceased and the P.C.V. had fallen to $50 \%$. A total of $850 \mathrm{ml}$. of plasma was given in the following 24 hours. At the time of the test the P.C.V. was $51 \%$ (plasma volume $71 \%$ of control). The control determination of antipyrine space was unsuccessful on two occasions, there being a technical fault with the first experiment and non-equilibration in the second experiment. The percentage fall in extracellular water and extracellular sodium was similar to that of the calculated plasma volume. The total body water value 48 hours after the burn was $52 \%$ body weight and equivalent to $141 . / \mathrm{m}^{2}{ }^{2}$ body surface area.

Case 4. A 9-year-old girl received $24 \%$ burns when lighting a gas cooker. The injury to the lower part of the trunk and both thighs included both full and partial thickness loss. During the first day $1,250 \mathrm{ml}$. plasma were given intravenously and the subsequent P.C.V. was $51 \%$. After $800 \mathrm{ml}$. of plasma administered during the second day, the P.C.V. had fallen to $47 \%$ (near to the control value of $44 \%$ ). The clinical condition was good, with a satisfactory urine output. The 
intracellular water was $92 \%$ of the control, and the extracellular water and the extracellular sodium had fallen to $77 \%$ and $73 \%$ respectively.

Case 5. After playing with matches, this 5-year-old girl sustained $17 \%$ burns of the gluteal region and thighs, mainly of a superficial nature. Plasma, $800 \mathrm{ml}$., was given intravenously during the first day and a P.C.V. of $39 \%$ was found. Next day $400 \mathrm{ml}$. plasma were given and when the test was performed the P.C.V. was $36 \%$ (control value $38 \%$ ). The patient was in a satisfactory clinical condition, with an adequate urine output and no vomiting or nausea. Total body water, intracellular and extracellular water, and $\mathrm{Na}$-extracellular water were increased; this was most marked in extracellular water $(154 \%)$ and extracellular sodium (140\%).

\section{B. Burns Without Specific Fluid Resuscitation Therapy}

Case 6. This patient was a girl of 18 months with $8 \%$ superficial burns of the left side of the trunk. After one day the P.C.V. was $37 \%$ and at the time of the test had attained the control value of $34 \%$. The clinical course was uneventful. Total body water was unchanged but there was an increase in extracellular water and extracellular sodium accompanied by a cellular water deficit.

Case 7. During the destruction of his home by fire, the patient, a 6-year-old boy, received $8 \%$ superficial burns of the trunk and arms. There was no clinical evidence of fluid deficit. At the time of the experimental measurements the P.C.V. $(41 \%)$ approximated to the control value $(39 \%)$. Total body water and intracellular water had fallen while the extracellular water and extracellular sodium showed an increase of approximately $10 \%$.

Case 8. A nightdress fire caused a 7\% superficial burn of the left arm and left side of the trunk in a 9-yearold girl. Her clinical condition was satisfactory. The P.C.V. at the end of the first day was $44 \%$, and at the time of the test $43 \%$. This represented $92 \%$ of the control plasma volume. The only change noted was a fall of $10 \%$ in intracellular water.

\section{Scalds Having Intravenous Resuscitation Therapy}

Case 9. This patient was a 21-month-old boy with a $10 \%$ superficial scald of the face and chest. Plasma, $400 \mathrm{ml}$., was given during the first 27 hours. The clinical course was uneventful, and when the tests were performed the P.C.V. was $40 \%$ (control value $38 \%$ ). A fall in extracellular water and extracellular sodium was accompanied by an increase in intracellular water with a slight increase in total body water.

Case 10. The patient, a boy of 2 years and 6 months, fell into a hot bath and sustained $12 \%$ superficial scalds of the left lower posterior trunk. During the first day $150 \mathrm{ml}$. plasma were transfused. Recovery was satisfactory. Extracellular water determination was unsuccessful. Total body water increased to $128 \%$ of the control value.

\section{Scalds Without Specific Fluid Resuscitation Therapy}

Case 11. A girl aged 21 months received $6 \%$ partial thickness scalds of the neck and shoulder when she spilled hot fat on to herself. The clinical recovery was satisfactory, and at the time of the experimental determination the P.C.V. was $37 \%$. The levels of the parameters were similar to the control values.

Case 12. A 22-month-old boy received a superficial hot water scald of the left shoulder and arm involving $4 \%$ of body surface. After 24 hours the P.C.V. was $39 \%$, and at 48 hours $37 \%$. The control value was $33 \%$. Clinical signs of distress were absent. Extracellular water and extracellular sodium were decreased while intracellular water remained constant.

Case 13. A boy aged 3 years and 8 months received a scald from spilled tea. The injury was superficial, covering $8 \%$ of the body surface (breast, abdomen, and genital region). The P.C.V. after 24 hours was $39 \%$, and $44 \%$ at the time of the experimental determinations. The control P.C.V. was $37 \%$, indicating a plasma volume $84 \%$ of the control value. Total body water was unchanged. The calculated increase in extracellular water was $71 \%$; although there was an undoubted increase $(p<0.05)$ the true extent of this lay within a wide possible range because of an excessive scatter of the experimental points.

Case 14. A 6-year-old girl was scalded when she spilled a bowl of hot inhalation fluid. The lower abdomen and perineal region sustained a superficial scald involving $8 \%$ of the body surface. The clinical condition was good. The P.C.V. and total body water were the same as the control values; extracellular water and extracellular sodium were reduced and intracellular water was correspondingly increased.

Response to Surgery. Similar estimations were made before operation (control experiment) and 46 hours after operation. There was the customary restriction of oral fluid for eight hours before and 24 hours afterwards, and no intravenous fluid therapy was given.

Case 15. A 5-year-old boy was admitted for surgical repair of the left hand three years after being burned. The P.C.V. after operation was $36 \%$, the control being $32 \%$. The experiments showed a marked fall in extracellular water and extracellular sodium, and a moderate fall in total body water, while the intracellular water was essentially unchanged.

Case 16. A 6-year-old boy was admitted for relief of a contracture of the left arm, resulting from burns sustained two years previously. At the time of the postoperative determinations the P.C.V. was $32 \%$, the control value being $35 \%$. Total body water was decreased, while extracellular water and extracellular sodium increased moderately, so that there was a considerable fall in intracellular water. 
TABLE 3

\begin{tabular}{|c|c|c|c|c|c|c|c|c|c|c|c|c|c|c|c|c|}
\hline \multirow{2}{*}{$\begin{array}{l}\text { Case } \\
\text { No. }\end{array}$} & \multirow[t]{2}{*}{ Sex } & \multirow{2}{*}{$\begin{array}{c}\text { Age } \\
\text { (yrs) }\end{array}$} & \multirow{2}{*}{$\begin{array}{l}\text { Weight } \\
\text { (kg.) }\end{array}$} & \multicolumn{2}{|c|}{ Iniury } & \multirow{2}{*}{$\begin{array}{l}\text { Replacement } \\
\text { Therapy } \\
\text { (ml.) }\end{array}$} & \multicolumn{2}{|c|}{$\underset{(\mathrm{mEq} / \mathrm{l} .)}{\text { Sodium }}$} & \multicolumn{2}{|c|}{$\begin{array}{l}\text { Potassium } \\
(\mathrm{mEq} / 1 .)\end{array}$} & \multicolumn{2}{|c|}{$\begin{array}{c}\text { Antipyrine } \\
\text { Space (litres) }\end{array}$} & \multicolumn{2}{|c|}{$\begin{array}{l}\text { Thiosulphate } \\
\text { Space (litres) }\end{array}$} & \multicolumn{2}{|c|}{ \% P.C.V. } \\
\hline & & & & $\begin{array}{l}\text { Surface } \\
\text { Area } \\
(\%)\end{array}$ & Type & & Acute & $\begin{array}{l}\text { Con- } \\
\text { trol }\end{array}$ & Acute & $\begin{array}{l}\text { Con- } \\
\text { trol }\end{array}$ & Acute & $\begin{array}{l}\text { Con- } \\
\text { trol }\end{array}$ & Acute & $\begin{array}{l}\text { Con- } \\
\text { trol }\end{array}$ & Acute & $\begin{array}{l}\text { Con- } \\
\text { trol }\end{array}$ \\
\hline 1 & $F$ & 8 & $20 \cdot 0$ & 21 & Burn & $\begin{array}{l}900 \text { plasma } \\
450 \text { Hartmann's sol. } \\
450 \text { saline }\end{array}$ & 135 & 153 & $4 \cdot 0$ & $4 \cdot 0$ & $10 \cdot 5$ & $12 \cdot 1$ & $2 \cdot 45$ & $2 \cdot 96$ & 47 & 40 \\
\hline 2 & $\mathbf{F}$ & 5 & $18 \cdot 7$ & 15 & Burn & $\begin{array}{l}500 \text { plasma } \\
100 \text { Hartmann's sol. }\end{array}$ & 130 & - & $3 \cdot 8$ & $3 \cdot 9$ & $9 \cdot 1$ & $9 \cdot 4$ & - & $2 \cdot 84$ & 43 & 36 \\
\hline $\begin{array}{l}3 \\
4 \\
5\end{array}$ & $\begin{array}{l}\mathbf{F} \\
\mathbf{F} \\
\mathbf{F}\end{array}$ & $\begin{array}{r}10 \\
9 \\
5\end{array}$ & $\begin{array}{l}30 \cdot 8 \\
26 \cdot 4 \\
18 \cdot 0\end{array}$ & $\begin{array}{l}10 \\
24 \\
17\end{array}$ & $\begin{array}{l}\text { Burn } \\
\text { Burn } \\
\text { Burn }\end{array}$ & $\begin{array}{l}850 \text { plasma } \\
2,250 \text { plasma } \\
1,200 \text { plasma }\end{array}$ & $\begin{array}{l}133 \\
135 \\
133\end{array}$ & $\begin{array}{l}140 \\
142 \\
146\end{array}$ & $\begin{array}{l}4 \cdot 5 \\
4 \cdot 5 \\
3 \cdot 8\end{array}$ & $\begin{array}{l}3 \cdot 9 \\
4 \cdot 0 \\
4 \cdot 0\end{array}$ & $\begin{array}{l}15 \cdot 9 \\
13 \cdot 0 \\
10 \cdot 8\end{array}$ & $\begin{array}{r}\overline{14 \cdot 8} \\
8 \cdot 9\end{array}$ & $\begin{array}{l}3 \cdot 21 \\
3 \cdot 27 \\
3 \cdot 34\end{array}$ & $\begin{array}{l}4 \cdot 23 \\
4 \cdot 25 \\
2 \cdot 16\end{array}$ & $\begin{array}{l}51 \\
47 \\
36\end{array}$ & $\begin{array}{l}36 \\
44 \\
38\end{array}$ \\
\hline $\begin{array}{l}6 \\
7 \\
8\end{array}$ & $\begin{array}{l}\mathbf{F} \\
\mathbf{M}\end{array}$ & $\begin{array}{l}1 \frac{1}{2} \\
6 \\
9\end{array}$ & $\begin{array}{r}9 \cdot 5 \\
19 \cdot 0 \\
21 \cdot 9\end{array}$ & $\begin{array}{l}8 \\
8 \\
7\end{array}$ & $\begin{array}{l}\text { Burn } \\
\text { Burn } \\
\text { Burn }\end{array}$ & E & $\begin{array}{l}140 \\
137 \\
137\end{array}$ & $\begin{array}{l}145 \\
140 \\
143\end{array}$ & $\begin{array}{l}3 \cdot 6 \\
3 \cdot 9 \\
4 \cdot 2\end{array}$ & $\begin{array}{l}4 \cdot 1 \\
4 \cdot 5 \\
3 \cdot 9\end{array}$ & $\begin{array}{r}4 \cdot 7 \\
9 \cdot 3 \\
12 \cdot 5\end{array}$ & $\begin{array}{r}4 \cdot 6 \\
10 \cdot 2 \\
13 \cdot 0\end{array}$ & $\begin{array}{l}1 \cdot 66 \\
2 \cdot 80 \\
2 \cdot 69\end{array}$ & $\begin{array}{l}1 \cdot 34 \\
2 \cdot 54 \\
2 \cdot 60\end{array}$ & $\begin{array}{l}34 \\
41 \\
43\end{array}$ & $\begin{array}{l}33 \\
39 \\
38\end{array}$ \\
\hline $\begin{array}{r}9 \\
10\end{array}$ & $\underset{\mathbf{F}}{\mathbf{M}}$ & $2 \frac{13}{4}$ & $\begin{array}{l}12 \cdot 0 \\
10 \cdot 6\end{array}$ & $\begin{array}{l}10 \\
12\end{array}$ & $\begin{array}{l}\text { Scald } \\
\text { Scald }\end{array}$ & $\begin{array}{l}400 \text { plasma } \\
150 \text { plasma }\end{array}$ & $\begin{array}{l}144 \\
145\end{array}$ & $\begin{array}{l}142 \\
139\end{array}$ & $\begin{array}{l}4 \cdot 0 \\
4 \cdot 0\end{array}$ & $\begin{array}{l}3 \cdot 9 \\
4 \cdot 0\end{array}$ & $\begin{array}{l}6 \cdot 3 \\
6 \cdot 8\end{array}$ & $\begin{array}{l}5 \cdot 9 \\
5 \cdot 3\end{array}$ & $1 \cdot 49$ & $1 \cdot 72$ & $\begin{array}{c}40 \\
(\mathrm{Hb} \\
9 \cdot 8 \\
\text { g. } \%)\end{array}$ & 38 \\
\hline $\begin{array}{l}11 \\
12 \\
13 \\
14\end{array}$ & $\begin{array}{l}\mathbf{F} \\
\mathbf{M} \\
\mathbf{M} \\
\mathbf{F}\end{array}$ & $\begin{array}{l}1 \frac{8}{4} \\
1 \frac{3}{4} \\
3 \frac{1}{2} \\
6\end{array}$ & $\begin{array}{l}11.6 \\
10.6 \\
16.8 \\
18.0\end{array}$ & $\begin{array}{l}6 \\
4 \\
8 \\
7\end{array}$ & $\begin{array}{l}\text { Scald } \\
\text { Scald } \\
\text { Scald } \\
\text { Scald } \\
\end{array}$ & E & $\begin{array}{l}148 \\
133 \\
145 \\
148\end{array}$ & $\begin{array}{l}138 \\
144 \\
148 \\
140\end{array}$ & $\begin{array}{l}3 \cdot 8 \\
4 \cdot 0 \\
3 \cdot 9 \\
3 \cdot 9\end{array}$ & $\begin{array}{l}3 \cdot 8 \\
3 \cdot 9 \\
3 \cdot 9 \\
4 \cdot 1\end{array}$ & $\begin{array}{l}5 \cdot 7 \\
6 \cdot 0 \\
9 \cdot 5 \\
9 \cdot 5\end{array}$ & $\begin{array}{l}5 \cdot 9 \\
6 \cdot 7 \\
9 \cdot 2 \\
9 \cdot 4\end{array}$ & $\begin{array}{l}1 \cdot 44 \\
1 \cdot 47 \\
5 \cdot 73 \\
2 \cdot 52\end{array}$ & $\begin{array}{l}1 \cdot 53 \\
2 \cdot 25 \\
3 \cdot 34 \\
3 \cdot 24\end{array}$ & $\begin{array}{l}37 \\
37 \\
44 \\
42\end{array}$ & $\begin{array}{l}39 \\
33 \\
37 \\
33\end{array}$ \\
\hline $\begin{array}{l}15 \\
16\end{array}$ & $\begin{array}{l}\mathbf{M} \\
\mathbf{M}\end{array}$ & $\begin{array}{l}5 \\
6\end{array}$ & $\begin{array}{l}20 \cdot 8 \\
21 \cdot 0\end{array}$ & - & - & - & $\begin{array}{l}134 \\
144\end{array}$ & $\begin{array}{l}135 \\
139\end{array}$ & $\begin{array}{l}3 \cdot 6 \\
3 \cdot 8\end{array}$ & $\begin{array}{l}4 \cdot 0 \\
3 \cdot 9\end{array}$ & $\begin{array}{r}8 \cdot 1 \\
10 \cdot 7\end{array}$ & $\begin{array}{r}8 \cdot 6 \\
12 \cdot 1\end{array}$ & $\begin{array}{l}2 \cdot 60 \\
3 \cdot 34\end{array}$ & $\begin{array}{l}3.34 \\
3.05\end{array}$ & $\begin{array}{l}36 \\
32\end{array}$ & $\begin{array}{l}32 \\
35\end{array}$ \\
\hline
\end{tabular}

Summary of Experimental Findings. After the burn or scald injury, changes in plasma sodium concentration were variable (Tables 3 and 4); in the majority the values lay within normal limits, but in six patients there was a moderate decrease. Plasma potassium concentration remained within the normal limits.

The estimated plasma volumes lay between $71 \%$ and $110 \%$ of the subsequent control values. Total body water and extracellular water ranged from $87 \%$ to $128 \%$, and $65 \%$ to $171 \%$ of the respective control values.
The half lives of thiosulphate and antipyrine, calculated from the rate of disappearance in the experiments, are shown in Table 6.

\section{Discussion}

1. Method. Parallel measurements of total body water in healthy children using deuterium oxide and antipyrine have given similar results (Friis-Hansen, Holliday, Stapleton and Wallace, 1951). In chronically ill adults with oedema, Hurst, Schemm and Vogel (1952) found that the deuterium oxide space

TABLE 4

\begin{tabular}{|c|c|c|c|c|c|c|c|c|c|c|c|c|}
\hline \multirow{2}{*}{$\begin{array}{l}\text { Case } \\
\text { No. }\end{array}$} & \multicolumn{2}{|c|}{ Antipyrine Space } & \multicolumn{2}{|c|}{ Thiosulphate Space } & \multicolumn{4}{|c|}{ Extracellular Sodium } & \multicolumn{3}{|c|}{ Calculated Intracellular Water } & \multirow[b]{2}{*}{$\begin{array}{c}\text { Ratio of } \\
\text { Plasma } \\
\text { Volumes } \\
\times 100\end{array}$} \\
\hline & $\begin{array}{c}\text { Differ- } \\
\text { ence } \\
\text { (litres) }\end{array}$ & $\frac{\text { Acute }}{\text { Control }} \times 100$ & $\begin{array}{c}\text { Differ- } \\
\text { ence } \\
\text { (litres) }\end{array}$ & Acute $\times 100$ & $\begin{array}{l}\text { Acute } \\
(\mathrm{mEq})\end{array}$ & $\begin{array}{c}\text { Control } \\
(\mathrm{mEq})\end{array}$ & $\begin{array}{c}\text { Differ- } \\
\text { ence } \\
\text { (mEq) }\end{array}$ & $\frac{\text { Acute }}{\text { Control }} \times 100$ & $\begin{array}{l}\text { Acute } \\
\text { (litres) }\end{array}$ & $\begin{array}{l}\text { Control } \\
\text { (litres) }\end{array}$ & $\frac{\text { Acute }}{\text { Control }} \times 100$ & \\
\hline $\begin{array}{l}1 \\
2 \\
3 \\
4 \\
5 \\
\end{array}$ & $\begin{array}{r}-1.6 \\
-0.3 \\
-1.8 \\
+1.9 \\
\end{array}$ & $\begin{array}{r}87 \\
97 \\
88 \\
121 \\
\end{array}$ & $\begin{array}{l}-0.5 \\
-1.0 \\
-1.0 \\
+1.2 \\
\end{array}$ & $\begin{array}{r}83 \\
76 \\
77 \\
154 \\
\end{array}$ & $\begin{array}{l}310 \\
395 \\
410 \\
410 \\
\end{array}$ & $\begin{array}{l}425 \\
545 \\
560 \\
295\end{array}$ & $\begin{array}{l}-115 \\
-150 \\
-100 \\
+115 \\
\end{array}$ & $\begin{array}{r}73 \\
73 \\
73 \\
74 \\
140 \\
\end{array}$ & $\begin{array}{r}8 \cdot 0 \\
12 \cdot 6 \\
9 \cdot 7 \\
7 \cdot 5 \\
\end{array}$ & $\begin{array}{r}9 \cdot 1 \\
6 \cdot 6 \\
10 \cdot 5 \\
6 \cdot 7 \\
\end{array}$ & $\begin{array}{r}\frac{88}{\overline{7}} \\
92 \\
112 \\
\end{array}$ & $\begin{array}{r}85 \\
84 \\
71 \\
94 \\
106 \\
\end{array}$ \\
\hline $\begin{array}{l}6 \\
7 \\
8\end{array}$ & $\begin{array}{l}-0.1 \\
-0.9 \\
-0.5\end{array}$ & $\begin{array}{l}98 \\
91 \\
96\end{array}$ & $\begin{array}{l}+0.3 \\
+0.3 \\
+0.1\end{array}$ & $\begin{array}{l}124 \\
110 \\
103 \\
\end{array}$ & $\begin{array}{l}215 \\
360 \\
340\end{array}$ & $\begin{array}{l}180 \\
330 \\
345\end{array}$ & $\begin{array}{l}+35 \\
+\quad 30 \\
-\quad 5\end{array}$ & $\begin{array}{r}120 \\
110 \\
99\end{array}$ & $\begin{array}{l}2 \cdot 9 \\
6 \cdot 5 \\
9 \cdot 8\end{array}$ & $\begin{array}{r}3 \cdot 4 \\
7 \cdot 7 \\
10 \cdot 4\end{array}$ & $\begin{array}{l}85 \\
85 \\
94\end{array}$ & $\begin{array}{l}97 \\
95 \\
88\end{array}$ \\
\hline $\begin{array}{r}9 \\
10 \\
\end{array}$ & $\begin{array}{r}+0.4 \\
+1.5 \\
\end{array}$ & $\begin{array}{l}107 \\
128 \\
\end{array}$ & $-0 \cdot 2$ & 87 & 200 & 230 & -30 & 87 & $4 \cdot 8$ & $\frac{4 \cdot 2}{-}$ & 114 & 95 \\
\hline $\begin{array}{l}11 \\
12 \\
13 \\
14 \\
\end{array}$ & $\begin{array}{l}-0.2 \\
-0.7 \\
+0.3 \\
+0.1 \\
\end{array}$ & $\begin{array}{r}97 \\
90 \\
103 \\
101 \\
\end{array}$ & $\begin{array}{l}-0.1 \\
-0.8 \\
+2.4 \\
-0.7\end{array}$ & $\begin{array}{r}94 \\
65 \\
171 \\
78 \\
\end{array}$ & $\begin{array}{l}200 \\
180 \\
770 \\
350\end{array}$ & $\begin{array}{l}200 \\
300 \\
460 \\
420\end{array}$ & $\begin{array}{r}0 \\
-120 \\
+310 \\
-\quad 70\end{array}$ & $\begin{array}{r}100 \\
60 \\
167 \\
83\end{array}$ & $\begin{array}{l}4 \cdot 3 \\
4 \cdot 5 \\
3 \cdot 8 \\
7 \cdot 0\end{array}$ & $\begin{array}{l}4 \cdot 4 \\
4 \cdot 4 \\
5 \cdot 9 \\
6 \cdot 2\end{array}$ & $\begin{array}{r}98 \\
102 \\
64 \\
113\end{array}$ & $\begin{array}{r}105 \\
89 \\
84 \\
79\end{array}$ \\
\hline $\begin{array}{l}15 \\
16\end{array}$ & $\begin{array}{l}-0.5 \\
-1.4\end{array}$ & $\begin{array}{l}94 \\
89\end{array}$ & $\begin{array}{l}-0 \cdot 7 \\
+0 \cdot 3\end{array}$ & $\begin{array}{r}78 \\
109\end{array}$ & $\begin{array}{l}320 \\
445\end{array}$ & $\begin{array}{l}420 \\
395\end{array}$ & $\begin{array}{l}-100 \\
+\quad 50\end{array}$ & $\begin{array}{r}77 \\
113\end{array}$ & $\begin{array}{l}5 \cdot 5 \\
7 \cdot 4\end{array}$ & $\begin{array}{l}5 \cdot 3 \\
9 \cdot 0\end{array}$ & $\begin{array}{r}104 \\
82\end{array}$ & $\begin{array}{r}89 \\
110\end{array}$ \\
\hline
\end{tabular}


TABLE 5

FLUID. SPACES MEASURED IN CONVALESCENCE

\begin{tabular}{|c|c|c|c|c|c|}
\hline \multirow{2}{*}{$\begin{array}{l}\text { Case } \\
\text { No. }\end{array}$} & \multirow{2}{*}{$\begin{array}{c}\text { Age } \\
\text { (yrs) }\end{array}$} & \multicolumn{2}{|c|}{ Extracellular Water } & \multicolumn{2}{|c|}{ Intracellular Water } \\
\hline & & $\mathrm{ml} . / 100 \mathrm{~g}$. & $1 . / \mathrm{m} .^{2}$ & $\mathrm{ml} . / 100 \mathrm{~g}$. & $1 . / \mathrm{m}^{2}$ \\
\hline $\begin{array}{r}1 \\
2 \\
3 \\
4 \\
5 \\
6 \\
7 \\
8 \\
9 \\
10 \\
11 \\
12 \\
13 \\
14 \\
15 \\
16\end{array}$ & $\begin{array}{c}5 \\
8 \\
10 \\
9 \\
5 \\
1 \frac{1}{2} \\
6 \\
9 \\
1 \frac{3}{4} \\
2 \frac{1}{2} \\
3 \frac{1}{2} \\
6 \\
2 \\
1 \frac{3}{4} \\
5 \\
6\end{array}$ & $\begin{array}{l}15 \\
15 \\
14 \\
16 \\
12 \\
14 \\
14 \\
12 \\
14 \\
13 \\
21 \\
20 \\
18 \\
16 \\
15\end{array}$ & $\begin{array}{l}3 \cdot 5 \\
3 \cdot 6 \\
3 \cdot 8 \\
4 \cdot 8 \\
2 \cdot 8 \\
3 \cdot 2 \\
3 \cdot 4 \\
3 \cdot 1 \\
3 \cdot 4 \\
-- \\
3 \cdot 6 \\
4 \cdot 3 \\
4 \cdot 8 \\
4 \cdot 5 \\
4 \cdot 3 \\
3 \cdot 8\end{array}$ & $\begin{array}{l}60 \\
49 \\
56 \\
49 \\
50 \\
54 \\
58 \\
49 \\
46 \\
51 \\
63 \\
55 \\
52 \\
41 \\
58\end{array}$ & $\begin{array}{l}14 \\
12 \\
17 \\
12 \\
11 \\
13 \\
16 \\
12 \\
13 \\
14 \\
13 \\
13 \\
13 \\
11 \\
15\end{array}$ \\
\hline Mean & & 15 & $3 \cdot 8$ & 53 & 13 \\
\hline
\end{tabular}

was approximately $10 \%$ greater than the antipyrine space. However, the two methods indicated changes of similar magnitude when total body water decreased as a result of therapy. Sodium thiosulphate has been used to measure the volume of extracellular water in children (Friis-Hansen, 1954), and in adults (Cardozo and Edelman, 1952). Nieth (1960) has found that the results correspond with inulin space measurements.

Thiosulphate enters cells so slowly under normal conditions (Cardozo and Edelman, 1952; Kowalski and Rutstein, 1952) that this is not a significant source of error in determining the extracellular water. It is, however, uncertain whether cellular permeability to the thiosulphate ion is altered as a result of thermal trauma, and therefore whether thiosulphate may overestimate the extracellular space. Cardozo and Edelman (1952) found that the increase of 'thiosulphate space' in oedematous patients was closely correlated with weight changes and clinical impressions.

In the present study, although some patients had well-marked clinical oedema, there was no evidence that the equilibration time for thiosulphate was increased. A similar situation obtained with the antipyrine measurement, where equilibration was always complete within 40 minutes with one exception (Case 3, control determination). This is at variance with the findings in oedematous adult patients by previous workers who have suggested equilibration times of more than five hours (Nieth, 1960).

Determinations of antipyrine and thiosulphate spaces in a group of healthy children have shown that there appears to be substantial variation at any given age (Friis-Hansen, 1956). In the current
TABLE 6

RATE OF REMOVAL OF THIOSULPHATE AND ANTIPYRINE FROM PLASMA

\begin{tabular}{|c|c|c|c|c|}
\hline \multirow{2}{*}{ Case } & \multicolumn{2}{|c|}{$\begin{array}{l}\text { Thiosulphate Half-life } \\
\text { (min.) }\end{array}$} & \multicolumn{2}{|c|}{$\begin{array}{c}\text { Antipyrine Half-life } \\
\text { (min.) }\end{array}$} \\
\hline & $\begin{array}{l}\text { After } \\
\text { Burn }\end{array}$ & $\begin{array}{c}\text { After } \\
\text { Convalescence }\end{array}$ & $\begin{array}{l}\text { After } \\
\text { Burn }\end{array}$ & $\begin{array}{c}\text { After } \\
\text { Convalescence }\end{array}$ \\
\hline $\begin{array}{r}1 \\
2 \\
3 \\
4 \\
5 \\
6 \\
7 \\
8 \\
9 \\
10 \\
11 \\
12 \\
13 \\
14\end{array}$ & $\begin{array}{l}\frac{15}{20} \\
16 \\
26 \\
25 \\
13 \\
18 \\
12 \\
\frac{18}{18} \\
13 \\
15\end{array}$ & $\begin{array}{l}18 \\
19 \\
19 \\
20 \\
14 \\
17 \\
12 \\
16 \\
17 \\
17 \\
30 \\
27 \\
20\end{array}$ & $\begin{array}{c}240 \\
\alpha^{*} \\
300 \\
200 \\
670 \\
380 \\
320 \\
430 \\
330 \\
240 \\
430 \\
\propto^{*} \\
230 \\
240\end{array}$ & $\begin{array}{l}420 \\
\propto * \\
910^{*} \\
150 \\
140 \\
170 \\
320 \\
150 \\
80 \\
200 \\
150 \\
270 \\
270\end{array}$ \\
\hline Mean & 18 & 19 & 334 & 210 \\
\hline
\end{tabular}

investigation, the values obtained during the acute post-burn phase were therefore compared with the convalescent values in order to remove as far as possible this source of variation. Such comparison of results is complicated by day-to-day and shortterm variations, the effects of which are unknown, but nevertheless is probably more valid than reference to values obtained in control subjects.

The extracellular water and total body water measurements determined on convalescent patients and expressed as a fraction of body weight and surface area are shown in Table 5 . The mean values are somewhat lower than those obtained by previous workers (Friis-Hansen et al., 1951; Friis-Hansen, 1954). Confidence intervals have been calculated for both antipyrine and thiosulphate dilution spaces; the $95 \%$ confidence intervals for 30 antipyrine space determinations averaged $\pm 5 \%$ of the values found, while 22 thiosulphate determinations had $95 \%$ confidence intervals of $\pm 8 \%$ of the dilution spaces found.

2. Rate of Disappearance of Thiosulphate and Antipyrine. Thiosulphate is removed from the blood principally by glomerular filtration, and also by movement into cells and subsequent metabolism. The results in Table 6 show no evidence of a fall in glomerular filtration rate in the acute phase, following these small degree burns and scalds. Only temporary changes in urea and creatinine clearances have been found in children (Graber and Sevitt, 1959), and a normal glomerular filtration rate is usually established in 24 to 72 hours.

The disappearance of antipyrine is mainly dependent on tissue metabolism (Soberman, Brodie, Levy, Axelrod, Hollander and Steele, 1949; Nieth, 
1960). In general the rate of disappearance of antipyrine is less in the acute post-burn phase, but this is not correlated with the severity of the burn. The half-life values determined in the control experiments agree with those in adults given by the workers quoted above, but in the acute post-burn period they are clearly increased, and this must be the result of disturbed metabolism.

3. Cases. The cases of Cope and Moore (1947), which were mainly treated with intravenous plasma and electrolyte solutions, showed an increase in extracellular water. This was observed in only one of five treated cases in the present series, while a similar increase was demonstrated in three untreated patients (Cases 6, 7 and 13) in which there was no increase of total body water. Three of four untreated scalds on the other hand showed a decrease in extracellular water (Cases 11, 12 and 14), two without significant change of intracellular water. This has previously been observed by Fox and Baer (1947), Rosenthal and Tabor (1945) and Fogelman and Wilson (1955). Since plasma sodium concentration showed no marked deviation in these untreated cases, total extracellular sodium varied pari passu with extracellular water.

Considering all cases, a majority showed a change in total body water in the same direction as changes in the calculated plasma volume. In treated burned patients the extracellular and intracellular spaces showed parallel increases or decreases of corresponding magnitude.

Except in Cases 1 and 2 (the former treated according to Evans, Purnell, Robinett, Batchelor and Martin, 1952), any intravenous fluid therapy was given solely as plasma. Case 3 was exceptional in that treatment was delayed for 24 hours, and had the lowest derived plasma volume after 46 hours, accompanied by an equally low extracellular water space and total extracellular sodium. Furthermore, on the third day there followed urticaria and ecchymoses which indicate capillary permeability changes additional to those usually occurring in burn injury.

Case 5 sustained a mainly superficial burn, whereas the other patients in this group suffered full thickness skin destruction. There was an expansion of the intracellular and extracellular spaces and although the amount of plasma given appeared excessive, this accumulation may be characteristic of superficial burns of large area. The magnitude of the increase in extracellular water is more than would be indicated from a consideration of the results of Cope and Moore (1947). In Case 9, which was not overcorrected with plasma, there was a similar increase in total body water accompanied by a fall in extracellular water and in Case 10, which is less well documented, an increase in total body water.

Cases 15 and 16, demonstrating post-operative response, showed a fall in total body water as a result of restriction of water intake. In Case 15, a child admitted for a minor operation, there was a decrease in extracellular space alone, while in Case 16 a decrease in intracellular space was accompanied by a moderate increase in extracellular space and total extracellular sodium. This may have been a result of post-operative adrenal activity, causing sodium retention and expansion of extracellular space. Sevitt (1954) has demonstrated adrenocortical hyperactivity following burns. However, the effect of this typical response to trauma on the distribution of fluid is difficult to assess since the situation is complicated by other changes. In four untreated cases (Cases 6, 7, 8 and 13) the results may be explained in this way.

4. Possible Clinical Significance. This study was deliberately restricted to children who had suffered thermal injuries of small extent. It had been noted during a review of the admissions to this burns unit that a few deaths had occurred in young children with burns or scalds involving only $5-10 \%$ of the body surface. Amongst this group five children aged 1-31 years developed acute central nervous disturbances with convulsions and died within one to 15 days of injury. At autopsy, two were found to have extensive cerebral venous thrombosis, another two showed cerebral swelling in the absence of any vascular accident and in the remaining instance no lesion of the brain was noted. Emery and Campbell-Reid (1962a, b) have also recorded the development of cerebral symptoms within two to eight days of relatively minor burns in seven children aged 3-13 months. Only one survived, with residual spastic hemiplegia; the others were found at autopsy to have gross cerebral swelling with pressure coning.

In the present study it was noted that unexpectedly large shifts of fluid within the body compartments sometimes occurred following burns or scalds of small extent. No relevant clinical disturbances were observed, but it is conceivable that such redistribution of fluid might on occasion be sufficient to provoke cerebral dysfunction.

\section{Conclusions}

The response to thermal injury is complex, because it results from the interplay of several factors. While the extent, depth and location of 
the skin involvement are important parameters, the effects of age and magnitude of capillary permeability changes are also relevant. Studies of the resulting disturbances are complicated in many instances by therapy. It is not to be expected, therefore, that a uniform pattern of response will be apparent.

Reduction in blood volume is clinically the most important early sequel of thermal injury, and this occurs even when the injury is of minor extent. The maintenance of a P.C.V. within limits which indicate a blood volume of $75-100 \%$ of the expected normal is of primary therapeutic importance. In parenthesis it may be noted that most of the cases reported here who received intravenous therapy were given plasma alone for the relief of oligaemia. Although some showed appreciable disturbances of fluid distribution on subsequent investigation, the clinical status of them and other cases treated in like manner in this burns unit suggests that the use of plasma alone for intravenous resuscitation may prove to be a simple and adequate mode of treatment, in the absence of appreciable red cell loss.

From this investigation of children with thermal injury involving less than $25 \%$ of the body surface, it appears that redistribution of fluid within the body varied not only in magnitude but also in direction. The changes were not correlated with the type of injury, its extent or the age of the patient.

The measurement of P.C.V. is not a reliable guide to changes which take place in body fluid compartments other than the vascular space. Moderate fluctuations in intra- and extracellular water are apparently tolerated in this type of stress situation. However, it seems that minor burns or scalds may on occasion provoke considerable redistribution of body fluid. It is therefore difficult to establish an arbitrary lower limit of percentage body surface involvement below which patients will not require intravenous fluid therapy, and this is in accordance with current clinical experience.

\section{Summary}

Simultaneous determinations of total body water and extracellular fluid (using antipyrine and thiosulphate respectively) were attempted in 14 children suffering from burns or scalds of small extent, and in two other children undergoing operation. The investigations were undertaken 48 hours after burn or operation, and repeated in convalescence to provide control values. The magnitude and direction of movement of body fluid were not correlated with the nature and extent of the trauma, nor with the age of the patients. It is of note, however, that considerable redistribution of fluid may result from burns or scalds that would be judged of minor nature from a clinical viewpoint.

\section{Appendix}

The colorimetric micromethod of Mendelson and Levin (1960) was used for analysis of antipyrine, modified to use $300 \mu$ l. of plasma. This enables two aliquots of deproteinized supernatant to be carried forward for analysis. To $300 \mu$ l. plasma are added $300 \mu \mathrm{l}$. 10.4\% cadmium sulphate $\left(3 \mathrm{CdSO}_{4} .8 \mathrm{H}_{2} \mathrm{O}\right)$ solution made up in $0.51 \overline{\mathrm{N}}$ sulphuric acid, followed by $300 \mu \mathrm{l}$. $\overline{\mathrm{N}}$ sodium hydroxide, and the mixture stirred with a glass rod. Centrifugation at $5,000 \mathrm{~g}$. for one minute yields enough supernatant to allow the subsequent analysis to be carried out in duplicate. In a dry test-tube $200 \mu \mathrm{l}$. supernatant are placed with $400 \mu \mathrm{l}$. glacial acetic acid, and $100 \mu 1.15 \%$ sodium nitrite are added. The solution is mixed by tapping, and after four minutes the nitrosation is stopped with $100 \mu 1.75 \%$ ammonium sulphamate. No shaking is required at this stage. Concentrated hydrochloric acid, $80 \mu 1$., and $0.5 \%$ naphthylethylenediamine dihydrochloride, $400 \mu \mathrm{l}$., are added. The tubes are then closed with silicone rubber bungs, inverted several times, and placed in a water bath at $80^{\circ} \mathrm{C}$. for 25 minutes. After cooling the tubes are again inverted several times and the absorbance measured at $580 \mathrm{~m} \mu$ in a Unicam SP600 spectrophotometer. Plasma and water blanks, and standards are treated similarly.

Thiosulphate was assayed by the method of Newman et al. (1946), modified to make use of plasma ultrafiltrate. This was prepared by means of a simplified version (Figure) of the apparatus described by Wilkinson (1960). A Lang-Levy pipette is used to add $200 \mu \mathrm{l}$. ultrafiltrate to a 5-ml. conical flask containing $1 \mathrm{ml}$. potassium iodate $(165 \mathrm{mg}$./litre) and two drops of $2 \overline{\mathrm{N}}$ hydrochloric acid. After standing for five minutes, four drops of freshly made $10 \%$ potassium iodide are added.

The liberated iodine is immediately titrated with $\frac{\mathrm{N}}{50}$ sodium thiosulphate delivered from a Conway microburette (capacity $250 \mu$ l.) using magnetic stirring. Two drops of $0.5 \%$ amylose are added near the end-point which is observed against an illuminated white background. In the presence of antipyrine the blue colour of amylose-iodine will return, and the true end-point is reached when the solution remains free of blue colour for two minutes. The titration is completed in five minutes.

A disappearance curve for thiosulphate was drawn by plotting the logarithm of the difference between titration of sample and of plasma blank against mid-time of sample collection. The co-ordinates of the experimental points were used to calculate the weighted regression line and intercept using the procedure described by Bennett and Franklin (1954).

The thiosulphate space $=\frac{(T-S)}{1} \times 125 \times V$,

where $T$ is blank titration; $S$ is titrations of $\frac{1}{125}$

injection solution; $I$ is antilogarithm of intercept at zero time; and $V$ is volume injected. 
Figure.-The ultrafiltration apparatus is constructed from two glass-flanged joints. The upper segment (A) is short and shaped to take a rubber cap. The lower segment (B) narrows into a tubulure. For use, a little silicone stopcock grease is applied to the flange of B, a surgeon's finger cot is stretched over the latter and unrolled down the barrel, and the flange placed in the lower member of the special clamp (Wilkinson, 1960). A 10-ml. syringe is connected to the tubulure via a stopcock, using silicone rubber tubing, and the plunger withdrawn to depress the rubber membrane. The plasma sample is placed in the cavity so formed and the pressure so adjusted that the surface of the plasma coincides with the plane of the flange. A square of 'cellophane' $(1 \cdot 5 \mathrm{in}$. $(3 \cdot 8 \mathrm{~cm}$.) (P.T.600, British Cellophane Ltd., Bridgewater, Somerset), which has been soaked in distilled water, is quickly blotted with filter paper and placed on the flange, avoiding as far as possible trapping air over the plasma. The upper segment (A) of the apparatus is placed in position, and the whole clamped firmly together.

The tubulure is now connected to a source of positive pressure (one atmosphere) overnight, during which time some $70 \%$ of the plasma water will collect in the upper compartment. Before removing the ultrafiltrate, it is advisable to disconnect from the source of positive pressure, to remove the risk of perforating the "cellophane."

A simple and convenient pressure reservoir and manifold can be made from a Buchner flask closed with a rubber bung which is penetrated by several lengths of nylon tubing; a bicycle tyre valve is secured to the side arm of the flask, and a bicycle pump used as a source of pressure. One of the nylon tubes is connected to a pressure gauge, and the others to the ultrafiltration units.

The antipyrine space was calculated using the method of least squares without weighting. The logarithm of the difference of absorbance between sample and plasma blank was plotted against mid-time of sample collection.

$$
\text { Antipyrine space }=\frac{(S-B)(1-P) \times 250 \times V,}{I}
$$

where $S$ is absorbance of $\frac{1}{250}$ injection solution; $\boldsymbol{B}$ is absorbance of water blank; $\boldsymbol{P}$ is protein fraction; and $I, V$, are as given above.

The method of antipyrine analysis used (Mendelson and Levin, 1960) was shown to be free of interference from thiosulphate ion, which is a disadvantage of the method of Brodie, Axelrod, Soberman and Levy (1949), according to Friis-Hansen et al. (1951). Plasma ultrafiltrate cannot be used for antipyrine determination; recovery varied between $70-90 \%$ with different samples of plasma, though the addition of glacial acetic acid (1-2 drops $/ \mathrm{ml}$.) reduced the $p \mathrm{H}$ to $4 \cdot 5-5 \cdot 0$ and improved the recovery to $90-95 \%$. There are different views with regard to the fixation of antipyrine to plasma proteins. Soberman et al. (1949) give the total binding of antipyrine as $10 \%$, whereas Schanker, Shore, Brodie and Hogben (1957) and Mendelson and Levin (1959) have found that there is no binding.

Initial attempts to recover sodium thiosulphate from solutions that had been ultrafiltered in the Wilkinson apparatus were unsuccessful because of the action of the mercury present. The apparatus was therefore modified to obviate this difficulty. The principal source of error in the titrimetric determination of thiosulphate is caused by loss of iodine from the solution. The use of microconical flasks, and the indicated quantity of potassium iodide, are essential to maintain reproducibility between titrations, and at the same time ensure observation of the end-point through a suitable depth of solution. On occasions 50 or $100 \mu l$. of ultrafiltrate have been used

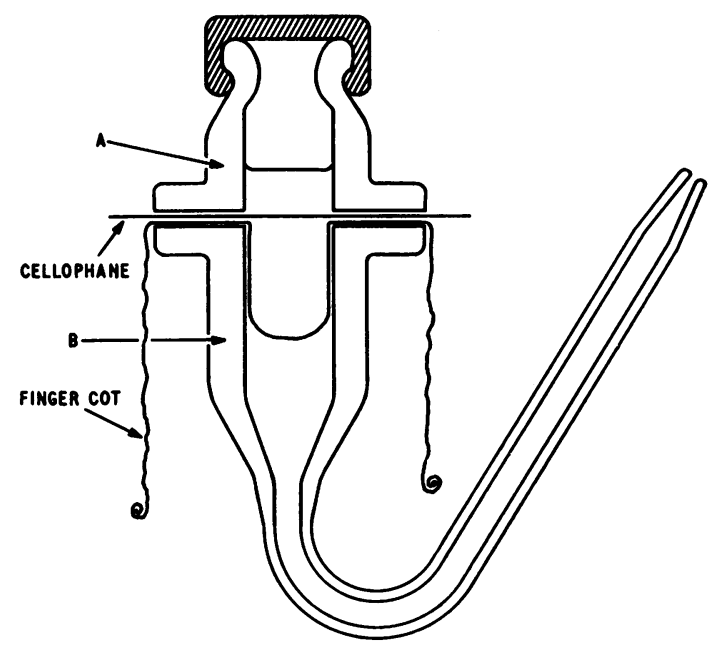

with appropriate dilution of reagents. The end-point is less easily seen but is satisfactory.

We should like to acknowledge our debt to Dr. L. L. R. White (Director of Patholøgy) for suggesting this investigation, and also for advice and criticism in the course of the work and the preparation of the manuscript.

We should also like to thank Mr. A. McDowall, F.R.C.S., and Mr. F. Robinson, F.R.C.S., the consultant surgeons to this burns unit, for their co-operation.

The study was made possible by a grant from the research fund of this hospital.

\section{REFERENCES}

Bennett, C. A. and Franklin, N. L (1954). Statistical Analysis in Chemistry and the Chemical Industry. Wiley, New York.

Brodie, B. B., Axelrod, J., Soberman, R. and Levy, B. B. (1949) The estimation of antipyrine in biological materials. J. biol. Chem., 179, 25 .

Bull, J. P. (1958). Nitrogen balance after injuries. Proc. Nutr. Soc., $17,114$.

Cope, O., Graham, J. B., Moore, F. D. and Ball, M. R. (1948). The nature of the shift of plasma protein to the extravascular
space following thermal trauma. Ann. Surg., 128, 1041. space following thermal trauma. Ann. Surg., 128, 1041 . in experimental burns and burn shock using radioactive dyes in blood and lymph. J. clin. Invest., 23, 241.

- (1947). The redistribution of body water and the fluid therapy of the burned patient. Ann. Surg., 126, 1010.

Cardozo, R. H and Edelman, I. S. (1952). The volume of distribution of sodium thiosulphate as a measure of extracellular fluid space. J. clin. Invest., 31, 280 .

Emery, J. L. and Campbell-Reid, C. (1962a). Swelling of the brain associated with minor burns. J. clin. Path., 15, 90. (1962b). Cerebral oedema and spastic hemiplegia following burns in young children. Brit. J. Surg., 50, 53.

Evans, E. I., Purnell, O. J., Robinett, P. W., Batchelor, A. and Martin, M. (1952). Fluid and electrolyte requirements in severe burns. Ann. Surg., 135, 804.

Fogelman, M. J. and Wilson, B. J. (1955). Blood, extracellular fluid, and total body water volume relationships in the early stages of severe burns. Surg. Forum, 5, 762.

Fox, C. L. and Baer, H. (1947). Redistribution of potassium, sodium, and water in burns and trauma, and its relation to the phenomena of shock. Amer. J. Physiol., 151, 155.

Friis-Hansen, B. (1954). The extracellular fluid volume in infants and children. Acta paediat. Scand., 43, 444.

(1956). Changes in body water compartments during growth. ibid., Suppl. 110. 
- Holliday, M. Stapleton, T. and Wallace, W. M. (1951). Total body water in children. Pediatrics, 7, 321 .

Graber, I. G. and Sevitt, S. (1959). Renal function in burned patients and its relationship to morphological changes. J. clin Path., 12, 25.

Hurst, W. W., Schemm, F. R. and Vogel, W. C. (1952). Simultaneous determination of total body water by antipyrine and deuterium oxide; evaluation of the methods on edematous subjects. J. Lab. clin. Med., 39, 36.

Kowalski, H. J. and Rutstein. D. D. (1952). The distribution of the thiosulphate ion with respect to normal human serum and red blood cells and its application to 'extracellular fluid' determination. J. clin. Invest., 31, 370.

Mendelson, D. and Levin, N. W. (1959). The estimation of total body water using a colorimetric method for the determination of antipyrine (1,5-dimethyl-2-phenyl-3-pyrazolone) in plasma. J. Lab. clin. Med., 54, 311 .

(1960). A colorimetric micromethod for the estimation of antipyrine in plasma or serum. S. Afr. J. med. Sci., 25, 13

Miles, A. A. and Wilhelm, D. L. (1960). The activation of endogenous substances inducing pathological increases in permeability. In The Biochemical Response to Injury, a Symposium, ed. H. B. Stoner and C. J. Threlfall, p. 51. Blackwell, Oxford.
Muir. J. and Stewart, F. (1959). Proceedings or the first full meeting held at the Mount Vernon Centre for plastic surgery, Northwood, May 2 .

Newman, E. V., Gilman, A and Philips, F. S. (1946). The renal clearance of thiosulphate in man. Johns Hopk. Hosp. Bull., 79, 229

Nieth, H. (1960). Plüssigkeiträume bei akuter und chronischer Niereninsuffizienz. Z. klin. Med., 156, 314.

Rosenthal, S. M. and Tabor, H. (1945). Electrolyte changes and chemotherapy in experimental burn and traumatic shock and hemorrhage. Arch. Surg., 51, 244.

Schanker, L. S. Shore, P. A., Brodie, B. B and Hogben, C. A. N (1957). Absorption of drugs from the stomach. J. Pharmacol. (Baltimore), $120,528$.

Sevitt, S. (1954). Adrenocortical function in burned patients; with special reference to A.C.T.H. and adrenaline tests. Brit. med.J., 1, 541 .

(1957). Burns: Pathology and Therapeutic Applications. Butterworth, London.

Soberman, R. J., Brodie, B. B., Levy, B. B., Axelrod, J., Hollander, V. and Steele, J. M. (1949). The use of antipyrine in the measurement of total body water in man. J. biol. Chem. 179, 31 .

Wilkinson, R. H. (1960). A micro ultrafiltration apparatus. J. clin. Path., 13, 268. 